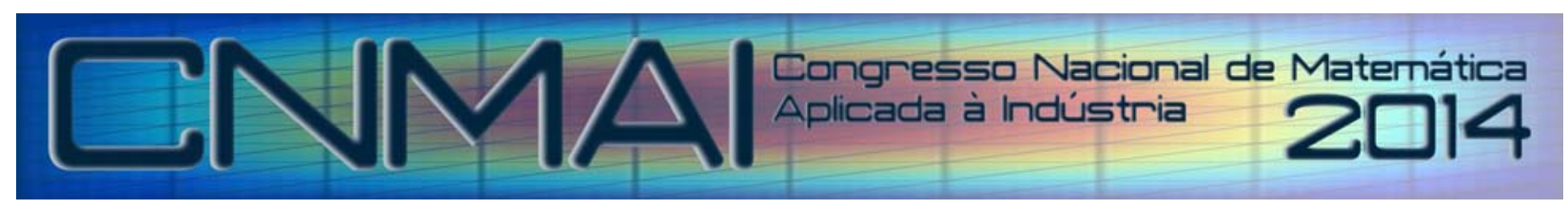

18 a 21 de novembro de 2014, Caldas Novas - Goiás

\title{
SISTEMA EÓLICO DE VELOCIDADE VARIÁVEL COM PMSG CONECTADO À REDE ELÉTRICA
}

\author{
Maro Jinbo, email: marojinbo@gmail.com ${ }^{1}$ \\ Ghendy Cardoso Junior, email: ghendy@ufsm.br ${ }^{1}$ \\ Felix Alberto Farret (IEEE Member), email: fafarret@gmail.com ${ }^{1}$ \\ Jordan Gustavo Trapp, email: jordantrapp@yahoo.com.br ${ }^{2}$ \\ Edson Ribeiro dos Santos, email:edsonrsmtm@gmail.com ${ }^{3}$ \\ Jawilson Pereira Machado, email: machado@uceff.edu.br ${ }^{4}$
}

\begin{abstract}
${ }^{1}$ Universidade Federal de Santa Maria (UFMS) - Departamento de Engenharia Elétrica, Santa Maria - RS, Brasil
${ }^{2}$ Instituto Federal Sul Rio-Grandense (IFSUL) - Departamento de Engenharia Elétrica, Venâncio Aires - RS - Brasil

${ }^{3}$ Universidade Federal da Fronteira Sul (UFFS) - Departamento de Matemática, Chapecó - SC, Brasil

${ }^{4}$ Faculdade Empresarial de Chapecó (UCEFF) - Depart. Eng. Ambiental e Sanitária, Chapecó - SC, Brasil
\end{abstract}

Resumo: Este artigo apresenta um Sistema Eólico de Velocidade Variável (VSWT Variable Speed Wind Turbine) com Gerador de Imãs Permanentes (PMSG Permanent Magnet Synchronous Generator). O PMSG é conectado à rede elétrica através de um conversor back-to-back. O modelo em Espaços de Estado em coordenadas dq0 do conversor trifásico bidirecional de três braços a IGBTs utilizados na retificação e inversão do sistema eólico será validado em simulação. Compensadores proporcional integral (PI) em coordenadas dq0 são utilizados no controle dos conversores. O projeto do compensador PI é executado através do software Matlab ${ }^{\circledR}$ e apresentado. Resultados de simulação através do software PSIM ${ }^{\circledR}$ tanto do retificador quanto do inversor são demonstrados a fim de comprovar as metodologias utilizadas e a eficácia do compensador projetado.

Palavras-chave: VSWT, PMSG, controle PI, conexão à rede.

\section{INTRODUÇÃO}

Sistemas de geração de energia baseados em fontes renováveis como a solar e a eólica estão se tornando cada vez mais importantes com o aumento percentual de suas contribuições na energia total gerada em certos países. Fatores como a escassez de potencial hidráulico a ser explorado, resíduos do uso da energia nuclear e a poluição que causa o carvão mineral fortalecem ainda mais a utilização de sistemas eólicos de energia (Salles, 2009).

Este artigo apresenta um sistema eólico de velocidade variável, conforme Fig. 1, o qual consiste em um gerador de ímãs permanentes conectado à rede elétrica através de um conversor $\mathrm{CA} / \mathrm{CC}$ (retificador) no lado do gerador e um conversor CC/CA (inversor) no lado da rede. Filtros $\mathrm{L}_{\mathrm{f}}$ são inseridos na linha do gerador e da rede para reduzir as harmônicas nas correntes geradas e injetadas. Os dois conversores são trifásicos com três braços, cada braço com duas chaves (IGBTs).

O controle do retificador trifásico em coordenadas dq0 utiliza dois compensadores do tipo PI (proporcionalintegral), um para o eixo direto e ou outro para o eixo em quadratura. Esse controle utiliza a velocidade do vento $(\mathrm{m} / \mathrm{s})$ para gerar uma referência de potência ativa através de uma tabela (loockup table) e um algoritmo MPPT (Maximum Power Point Tracking) realiza o rastreamento do ponto de máxima potência.

Os compensadores do controle do inversor trifásico também são do tipo PI e tem a função de controlar as correntes trifásicas injetadas na rede de acordo com a potência ativa que o gerador entrega ao retificador. Além disso, uma malha de tensão contribui na referência de potência ativa injetada na rede, controlando a tensão sobre o barramento CC.

Considera-se em ambos os lados, gerador e rede elétrica, fator de potência unitário. 


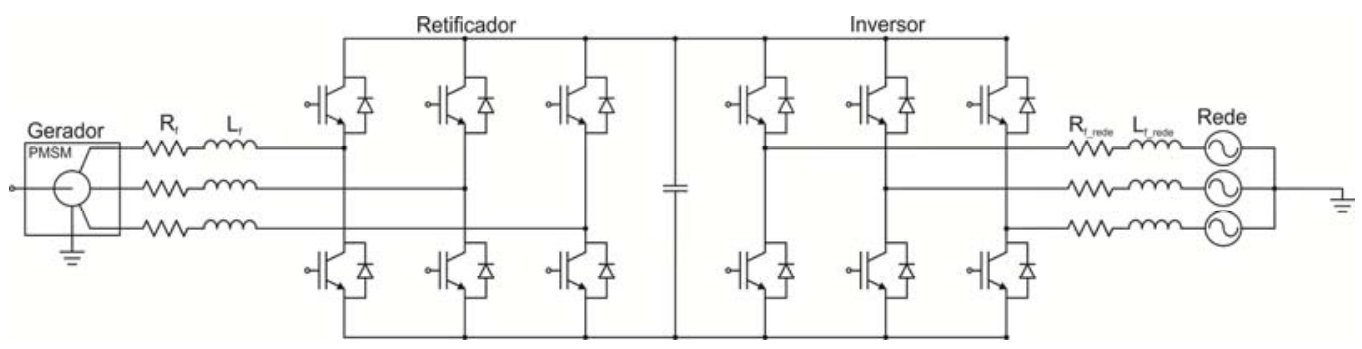

Figura 1. Sistema eólico VSWT conectado à rede.

\section{MODELAGEM DO CONVERSOR ESTÁTICO}

A modelagem do conversor trifásico bidirecional (Borgono, 2005 e Collier, 2011) é realizada neste artigo. O modelo é utilizado para a análise e projeto de controladores para os dois conversores do sistema eólico através da utilização da ferramenta Sisotool do Matlab ${ }^{\circledR}$. O modelo da Fig. 2 considera algumas simplificações, como por exemplo, a fonte trifásica senoidal ideal $\mathrm{V}_{\mathrm{a}}, \mathrm{V}_{\mathrm{b}}$ e $\mathrm{V}_{\mathrm{c}}$ e a tensão no barramento $C C$ igual a uma tensão contínua $\mathrm{V}_{\mathrm{dc}}$ resultando nas equações apresentadas na Eq. (1).

\subsection{Modelo em Espaço de Estados - Coordenadas abc}

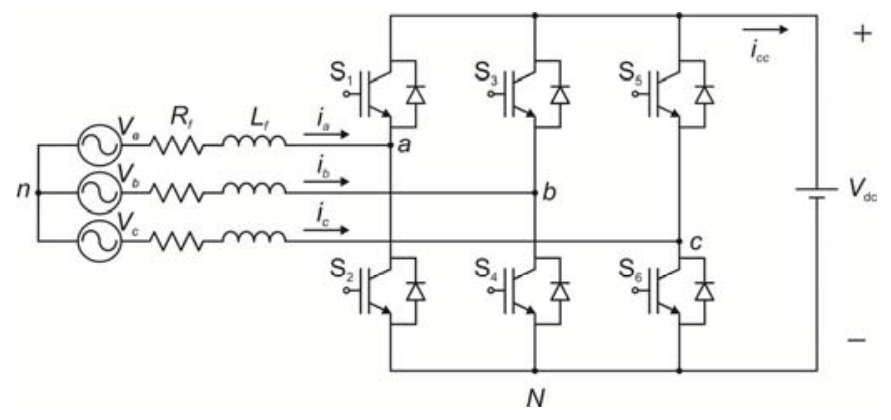

Figura 2. Conversor estático bidirecional.

$$
\begin{aligned}
& V_{a}=R_{f} i_{a}+L_{f} \dot{i}_{a}+u_{a P W M}+V_{N n} \\
& V_{b}=R_{f} i_{b}+L_{f} \dot{i}_{b}+u_{b P W M}+V_{N n} \\
& V_{c}=R_{f} i_{c}+L_{f} \dot{i}_{c}+u_{c P W M}+V_{N n}
\end{aligned}
$$

A diferença de potencial $\mathrm{u}_{\mathrm{xPWM}}$ é a tensão entre o ponto $\mathrm{x}(\mathrm{x}=\mathrm{a}$ ou $\mathrm{b}$ ou c) e o ponto $\mathrm{N}$ da Fig. 2. Somando as três equações Eq. (1), aplicando-se a Lei de Kirchoff das correntes no nó $\mathrm{n}$ e realizando-se as devidas substituições obtêm-se as equações em espaços de estados do conversor bidirecional em coordenadas abc, conforme Eq. (2) (Borgono, 2005 e Collier, 2011). A tensão $\mathrm{u}_{\mathrm{xPWM}}$ é substituída na Eq. (2) pelo produto da tensão no barramento CC e a razão cíclica, $\mathrm{V}_{\mathrm{dc}} \cdot \mathrm{d}_{\mathrm{x}}(\mathrm{t})$ resultando na Eq. (3);

$$
\begin{gathered}
{\left[\begin{array}{l}
\dot{i_{a}} \\
\dot{i_{b}} \\
\dot{i_{c}}
\end{array}\right]=\frac{R_{f}}{L_{f}}\left[\begin{array}{ccc}
-1 & 0 & 0 \\
0 & -1 & 0 \\
0 & 0 & -1
\end{array}\right]\left[\begin{array}{l}
i_{a} \\
i_{b} \\
i_{c}
\end{array}\right]+\frac{1}{3 L_{f}}\left[\begin{array}{ccc}
-2 & 1 & 1 \\
1 & -2 & 1 \\
1 & 1 & -2
\end{array}\right]\left[\begin{array}{l}
u_{a P W M} \\
u_{b P W M} \\
u_{c P W M}
\end{array}\right]+\frac{1}{3 L_{f}}\left[\begin{array}{ccc}
-2 & -1 & -1 \\
-1 & 2 & -1 \\
-1 & -1 & 2
\end{array}\right]\left[\begin{array}{c}
V_{a} \\
V_{b} \\
V_{c}
\end{array}\right]} \\
{\left[\begin{array}{l}
\dot{i_{a}} \\
\dot{i_{b}} \\
\dot{i_{c}}
\end{array}\right]=\frac{R_{f}}{L_{f}}\left[\begin{array}{ccc}
-1 & 0 & 0 \\
0 & -1 & 0 \\
0 & 0 & -1
\end{array}\right]\left[\begin{array}{l}
i_{a} \\
i_{b} \\
i_{c}
\end{array}\right]+\frac{V_{d c}}{3 L_{f}}\left[\begin{array}{ccc}
-2 & 1 & 1 \\
1 & -2 & 1 \\
1 & 1 & -2
\end{array}\right]\left[\begin{array}{l}
d_{a P W M} \\
d_{b P W M} \\
d_{c P W M}
\end{array}\right]+\frac{1}{3 L_{f}}\left[\begin{array}{ccc}
-2 & -1 & -1 \\
-1 & 2 & -1 \\
-1 & -1 & 2
\end{array}\right]\left[\begin{array}{l}
V_{a} \\
V_{b} \\
V_{c}
\end{array}\right]}
\end{gathered}
$$


Ou na forma padrão, Eq. (4) e Eq. (5):

$$
\begin{gathered}
\dot{X(t)}=A_{a b c} X(t)+B_{a b c} d(t)+F_{a b c} W(t) \\
Y(t)=C_{a b c} X(t)+E_{a b c} U(t)
\end{gathered}
$$

onde:

$\mathrm{X}(\mathrm{t})$ é o vetor de estados, as correntes nos indutores $\mathrm{L}_{\mathrm{f}}$;

$\mathrm{u}(\mathrm{t})$ é o vetor de controle, as tensões em cada braço;

$\mathrm{d}(\mathrm{t})$ é o vetor com as razões cíclicas $\left(\mathrm{S}_{1} \mathrm{~S}_{3} \mathrm{~S}_{5}\right)$;

$\mathrm{W}(\mathrm{t})$ é o vetor de distúrbios nas tensões da fonte $\mathrm{CA}$;

$\mathrm{Y}(\mathrm{t})$ é o vetor saída, correntes nos indutores $\mathrm{I}_{\mathrm{a}}, \mathrm{I}_{\mathrm{b}}$ e $\mathrm{I}_{\mathrm{c}}$;

$\mathrm{U}(\mathrm{t})$ é o vetor entrada, $\mathrm{d}_{\mathrm{a}}, \mathrm{d}_{\mathrm{b}}, \mathrm{d}_{\mathrm{c}}, \mathrm{V}_{\mathrm{a}}, \mathrm{V}_{\mathrm{b}}$ e $\mathrm{V}_{\mathrm{c}}$.

\subsection{Modelo em Espaço de Estados - Coordenadas dq0}

Realizando-se a transformação de Park, o qual transforma as coordenadas abc em coordenadas dq0 através da matriz T ${ }^{-1}$ apresentada na Eq. (6), obtém-se o modelo em espaços de estados do conversor em coordenadas dq0, Eq. (7) e Eq. (8):

$$
\begin{gathered}
T^{-1}=\left[\begin{array}{ccc}
\frac{\sqrt{6}}{3} \cos (\theta) & -\frac{\sqrt{6}}{6} \cos (\theta)+\frac{\sqrt{2}}{2} \operatorname{sen}(\theta) & -\frac{\sqrt{6}}{6} \cos (\theta)-\frac{\sqrt{2}}{2} \operatorname{sen}(\theta) \\
-\frac{\sqrt{6}}{3} \operatorname{sen}(\theta) & \frac{\sqrt{6}}{6} \operatorname{sen}(\theta)+\frac{\sqrt{2}}{2} \cos (\theta) & \frac{\sqrt{6}}{6} \operatorname{sen}(\theta)-\frac{\sqrt{2}}{2} \cos (\theta) \\
\frac{\sqrt{3}}{3} & \frac{\sqrt{3}}{3} & \frac{\sqrt{3}}{3}
\end{array}\right] \\
\dot{X}_{d q 0(t)}=A_{d q 0} X_{d q 0}(t)+B_{d q 0} d_{d q 0}(t)+F_{d q 0} W_{d q 0}(t) \\
Y(t)_{d q 0}=C_{d q 0} X(t)_{d q 0}+E_{d q 0} U(t)_{d q 0}
\end{gathered}
$$

onde:

$$
\begin{aligned}
X_{d q 0} & =\left[\begin{array}{c}
i_{d} \\
i_{q} \\
0
\end{array}\right] \\
A_{d q 0}=T^{-1}\left(A_{a b c} T-\dot{T}\right) & =\left[\begin{array}{ccc}
-\frac{R_{f}}{L_{f}} & \omega & 0 \\
-\omega & -\frac{R_{f}}{L_{f}} & 0 \\
0 & 0 & -\frac{R_{f}}{L_{f}}
\end{array}\right] \\
B_{d q 0}=T^{-1} B_{a b c} T & =\frac{V_{d c}}{L_{f}}\left[\begin{array}{ccc}
-1 & 0 & 0 \\
0 & -1 & 0 \\
0 & 0 & 0
\end{array}\right]
\end{aligned}
$$




$$
F_{d q 0}=T^{-1} F_{a b c} T=\frac{1}{L_{f}}\left[\begin{array}{lll}
1 & 0 & 0 \\
0 & 1 & 0 \\
0 & 0 & 0
\end{array}\right]
$$

\subsection{Funções de Transferências do Conversor - Planta}

A partir da Eq. (7), modelo em espaço de estados do conversor em coordenadas dq0, pode-se extrair as funções de transferências da planta para o projeto dos controladores. Para isso, pode-se utilizar a função ss2tf disponível no Matlab $^{\circledR}$. Assim, obtêm-se as funções de transferências das saídas $i_{d}$ e $i_{q}$ em relação a cada uma das entradas $d_{d}, d_{q}, V_{d} e$ $\mathrm{V}_{\mathrm{q}}$ de forma literal ou numérica. As funções de interesse são $\mathrm{i}_{\mathrm{d}} / \mathrm{d}_{\mathrm{d}}$ Eq. (13) e $\mathrm{i}_{\mathrm{q}} / \mathrm{d}_{\mathrm{q}}$ Eq. (14) para a análise e projeto de controladores. As equações Eq. (13) e Eq. (14) são idênticas neste caso.

Função de transferência da corrente $i_{d}$ em função de $d_{d}$ :

$$
\frac{i_{d}}{d_{d}}=\frac{V_{c c} L_{f} s+V_{c c} R_{f}}{L_{f}{ }^{2} s^{2}+2 L_{f} R_{f} s+\left(R_{f}{ }^{2}+L_{f}{ }^{2} \omega^{2}\right)}
$$

Função de transferência da corrente $\mathrm{i}_{\mathrm{q}}$ em função de $\mathrm{d}_{\mathrm{q}}$ :

$$
\frac{i_{q}}{d_{q}}=\frac{V_{c c} L_{f} s+V_{c c} R_{f}}{L_{f}^{2} s^{2}+2 L_{f} R_{f} s+\left(R_{f}^{2}+L_{f}^{2} \omega^{2}\right)}
$$

\section{CONTROLE}

\subsection{Controle do Conversor no Lado do Gerador PMSG}

O controle do conversor no lado do gerador (Muyeen; Takahashi; Ali, 2007 e Muyeen; Takahashi; Murata, 2008) é responsável por realizar a transferência de energia para o barramento cc baseado nas referências de potência ativa P. A referência $\mathrm{P}$ é oriunda de um valor de potência ativa de uma tabela (lookup table) que relaciona velocidade do vento em $\mathrm{m} / \mathrm{s}$ e potência equivalente em Watts. Este valor tabelado de potência é processado em um algoritmo MPPT (Maximum Power Point Tracker) (Errami, 2012) que resulta em $\mathrm{P}^{*}$ aplicado como referência de potência ativa no controle do retificador. A referência de potência reativa $\mathrm{Q}^{*}$ é ajustada em zero no controle do retificador para um fator de potência unitário. O diagrama de blocos do controle do conversor do lado do gerador está ilustrado na Fig. 3. C1 e C2 são os compensadores do controle deste estágio. Efetivamente o controle atua nas correntes de linha geradas $\mathrm{I}_{\mathrm{abc}}$ na saída do gerador, mas em coordenadas dq0 utilizadas no controle são as correntes $\mathrm{I}_{\mathrm{dq} 0}$.

As referências de correntes geradas $\mathrm{i}_{\mathrm{d}}{ }^{*}$ e $\mathrm{i}_{\mathrm{q}}{ }^{*}$ estão de acordo com a Eq. (15) e Eq. (16).

$$
\begin{aligned}
& i_{d}^{*}(t)=\frac{P^{*} \cdot V_{d}+Q^{*} \cdot V_{q}}{V_{d}^{2}+V_{q}^{2}} \\
& i_{q}^{*}(t)=\frac{P^{*} \cdot V_{q}-Q^{*} \cdot V_{d}}{V_{d}^{2}+V_{q}^{2}}
\end{aligned}
$$

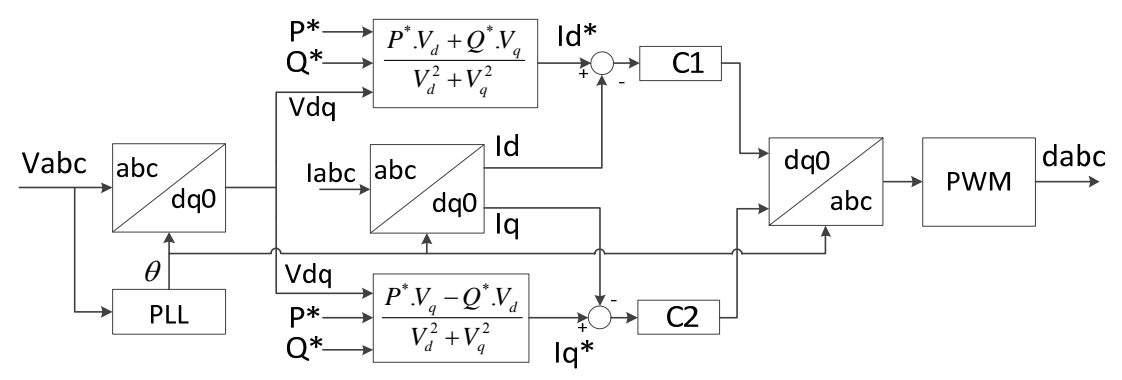

Figura 3. Diagrama de controle do retificador. 


\subsection{Controle do Conversor no Lado da Rede Elétrica}

O controle do conversor no lado da rede elétrica (Nascimento, 2009), apresentado na Fig. 4 e Fig. 5, tem a função de controlar as correntes injetadas na rede $\mathrm{I}_{\mathrm{abc}}$ ou $\mathrm{I}_{\mathrm{dq} 0}$ de acordo com as referências de potência ativa $\mathrm{P}^{*}$ rede $\mathrm{e}$ reativa $\mathrm{Q}^{*}$ rede. Na Fig. 5 temos a $\mathrm{P}_{\text {transferida }}$ que é a potência ativa que o gerador efetivamente transfere para o retificador e consequentemente ao barramento cc. Enquanto $\mathrm{Q}^{*}{ }_{\text {rede }}$ é a potência reativa demandada pela rede que neste artigo foi ajustado em $0 \mathrm{VAr}$, ou seja, fator de potência unitário. A tensão no barramento $\mathrm{V}_{\mathrm{dc}}$ é regulada através da referência de tensão $\mathrm{V}_{\mathrm{dc}}{ }^{*}$, e esta diferença de tensão multiplicada pela corrente $\mathrm{I}_{\mathrm{dc}}$ que entra no inversor contribui com acréscimos e descrécimos de potência ativa na referência de $\mathrm{P} *_{\text {rede. }}$

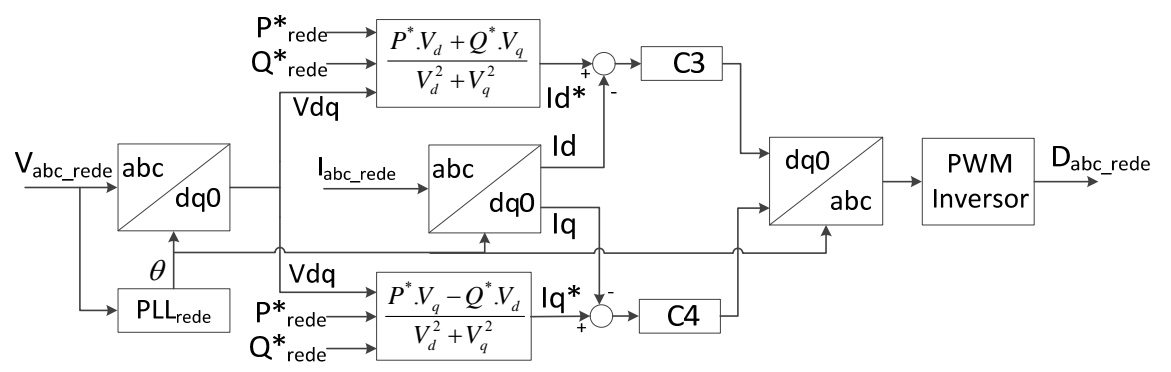

Figura 4. Diagrama de controle do inversor.

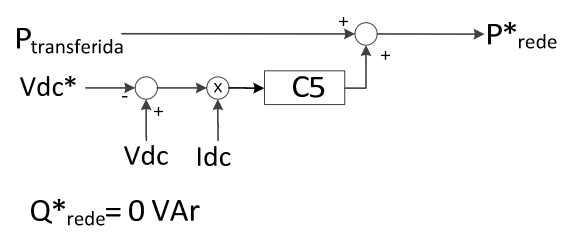

Figura 5. Referências de potências do controle do inversor.

\section{PROJETO DE COMPENSADORES}

\subsection{Compensador da Malha de Corrente}

O controle do conversor no lado do gerador PMSG em coordenadas dq0 (Li, 2009 e Krishnan, 2010) possui dois compensadores, $\mathrm{C} 1$ no eixo d e C2 no eixo q, de acordo com o diagrama da Fig. 3. Os compensadores C3 e C4 são referentes aos eixos d e q do controle do conversor no lado da rede, como apresentado na Fig. 4 (Barbi; Martins, 2005). Na Fig. 5 ainda temos um compensador C5 do tipo P (proporcional) que atua na regulação da tensão no barramento cc. Esses compensadores serão objeto de projeto, análise e simulação.

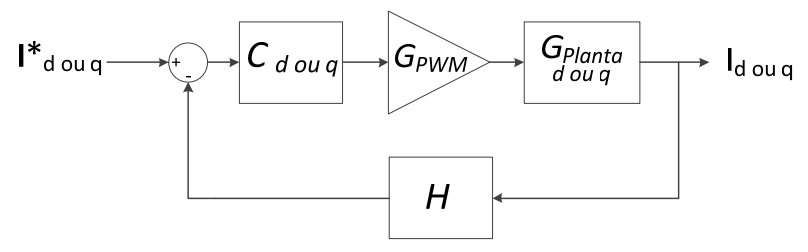

Figura 6. Diagrama de controle em coordenadas d e q.

Na Figura 6 temos o diagrama de blocos do controle das correntes $i_{d}$ e $i_{q}$ que consistem nas correntes da saída do gerador ou as correntes injetadas na rede em coordenadas dq0. As correntes $i_{d}{ }^{*}$ e $i_{q}{ }^{*}$ são as referências de correntes conforme Fig. 3 e Fig. 4.

$\mathrm{C}_{\mathrm{d} \text { ou q }}$ faz referência a um dos quatro compensadores que serão projetados $(\mathrm{C} 1, \mathrm{C} 2, \mathrm{C} 3$ ou $\mathrm{C} 4)$. $\mathrm{G}_{\mathrm{PWM}}$ é o ganho referente ao ajuste de amplitude do sinal modulante em relação à portadora. $\mathrm{O}$ ganho $\mathrm{H}$ da realimentação será considerado de valor unitário. $G_{\text {Planta }}$ é a função de transferência apresentada na Eq. (13) e Eq. (14).

O projeto do compensador C3 do inversor será efetuado detalhadamente. A Tabela 1 apresenta as especificações do conversor utilizado. 
Tabela 1. Especificações do inversor.

\begin{tabular}{cc}
\hline Tensão de linha da rede & $127 \mathrm{Vrms}$ \\
\hline Frequência da rede & $60 \mathrm{~Hz}$ \\
\hline Tensão no barramento $\mathrm{CC}$ & $350 \mathrm{~V}$ \\
\hline Resistência do filtro $\mathrm{R}_{\mathrm{f}}$ & $0.12 \Omega$ \\
\hline Indutância do filtro $\mathrm{L}_{\mathrm{f}}$ & $2.5 \mathrm{mH}$ \\
\hline Frequência de Chaveamento $\mathrm{f}_{\mathrm{s}}$ & $12 \mathrm{KHz}$ \\
\hline
\end{tabular}

Utilizando-se o Matlab $^{\circledR}$ e aplicando-se os valores da Tab. 1 na Eq. (7) obtém-se a seguinte função de transferência:

$$
\frac{i_{d}}{d_{d}}=\frac{-75,68 s^{2}-7265 s-1,744.10^{5}}{s^{3}+144 s^{2}+1,49.10^{5} s+6,932.10^{6}}
$$

Utilizando-se a Eq. (17) como $\mathrm{G}_{\text {Planta_d }}$, multiplicando-se por $\mathrm{G}_{\mathrm{PWM}}=1$ de acordo com a Fig. 6, obtem-se $\mathrm{G}_{\text {Planta_d }}$ $\mathrm{xG}_{\mathrm{PWM}}$. Este produto consiste na função de transferência em malha aberta do sistema de controle. O diagrama de Bode do sistema não compensado apenas com a planta $\mathrm{G}_{\text {Planta d, }}$, Fig. 7.

O projeto do compensador C3 foi realizado utilizando-se a ferramenta SISOTOOL do Matlab. Objetivou-se o projeto de um compensador do tipo PI, bastante comum neste tipo de aplicação.

Pode-se observar na Fig. 7 que a planta possui uma margem de fase de $-90^{\circ}$ e uma frequência de cruzamento $f_{c}=$ $22,3 \mathrm{KHz}$ bem acima da frequência de chaveamento $\mathrm{f}_{\mathrm{s}}=12 \mathrm{KHz}$. Dessa forma, projetou-se um compensador PI, objetivando uma frequência de cruzamento $f_{c}=f_{s} / 10$ uma década abaixo da frequência de chaveamento ou mais próximo possível. O que resultou em uma margem de fase de $88,2^{\circ}$, malha estável, $\mathrm{f}_{\mathrm{c}}=2,23 \mathrm{KHz}$ como pode ser observado no diagrama de bode do sistema compensado apresentado na Fig. 8.

$$
C_{3}=-50 \frac{(1+0.002 s)}{s}
$$

Considerando-se a utilização do mesmo modelo matemático em espaço de estados para o retificador e também para o inversor, adotou-se consequentemente o mesmo compensador Eq. (18) nos demais compensadores C1, C2 e C4.

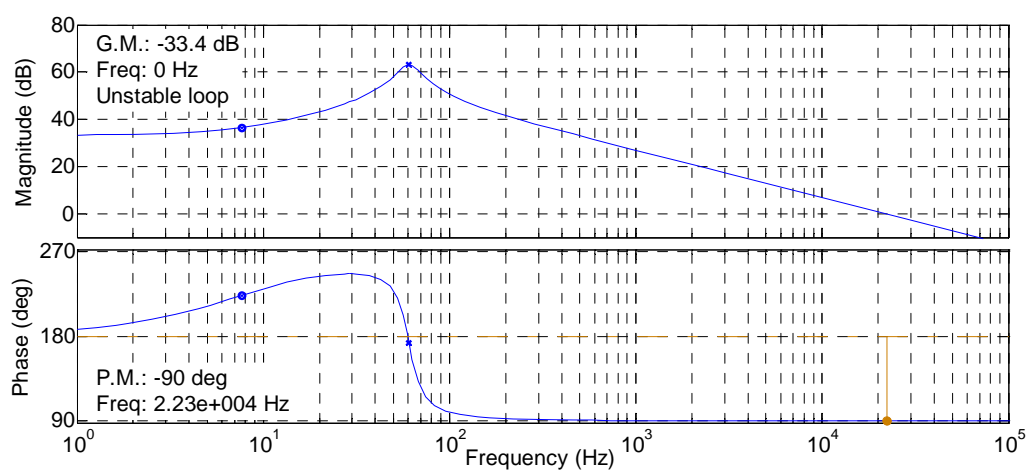

Figura 7. Diagrama de Bode $G_{\text {Planta_d. }}$

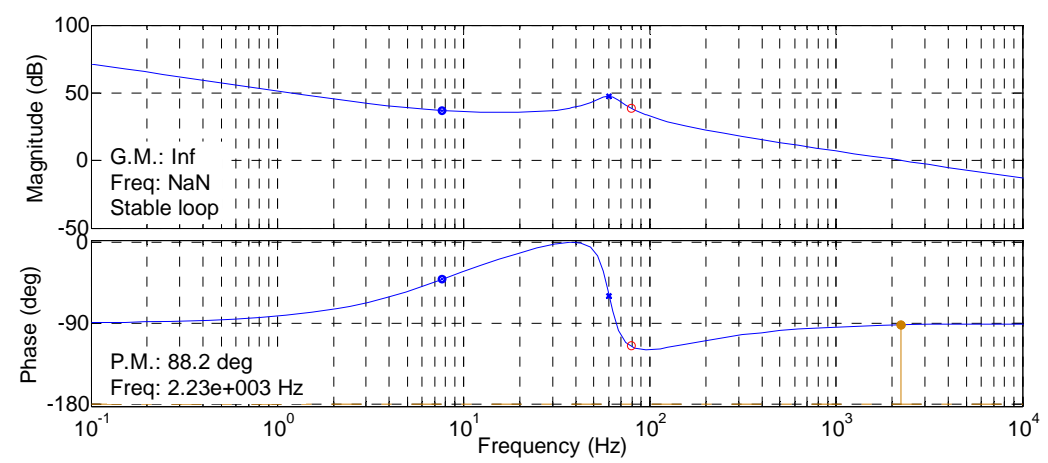

Figura 8. Diagrama de Bode da Planta Compensada. 


\subsection{Compensador da Malha de Tensão do Inversor}

A regulação da tensão no barramento $\mathrm{cc}$ em $\mathrm{V}_{\mathrm{dc}}=350 \mathrm{~V}$ é realizado de acordo com o diagrama da Fig. 5. A potência ativa de referência $\mathrm{P}^{*}$ rede é a composição entre a potência ativa que o gerador transfere para o retificador denominado de $\mathrm{P}_{\text {transferida }}$ que é medido no controle do retificador e a parcela proveniente da regulação da tensão no barramento.

O compensador C5 da Fig. 5 é do tipo P (proporcional), cujo valor é obtido pelo balanço entre as potências ativas entregue a rede e as potências no barramento cc, valor ajustado conforme Eq. (19) a seguir:

$$
C_{5}=0.5
$$

\section{SIMULAÇÃO}

As simulações foram realizadas inicialmente no Simulink/Matlab ${ }^{\circledR}$ para demonstrar a validação das equações em espaço de estados do conversor bidirecional Eq. (7). Esta etapa de simulação foi realizada somente para o conversor operando como inversor, injetando correntes na rede elétrica. Correntes $i_{\mathrm{dq} \text { rede }}$ do circuito e do modelo foram sobrepostos na visualização, Fig. 9. Na etapa seguinte de simulações com PSIM $^{\mathbb{R}}$ validou-se o modelo também para o conversor operando como retificador.

A segunda parte das simulações foram realizadas no $P S I M^{\circledR}$ com o sistema eólico interligado, gerador $P M S G$, retificador, inversor e a rede elétrica. Apresenta-se o funcionamento do sistema eólico, através das correntes geradas e injetadas, tensão no barramento $\mathrm{CC}$, velocidade de rotação do gerador, sinais de controle seguindo suas referências tanto no controle do retificador como do inversor entre outros sinais. Pretende-se demonstrar o desempenho dos compensadores projetados.

\subsection{Simulação do Modelo em Espaço de Estados dq0}

A simulação de validação do modelo em espaço de estados em coordenadas dq0, Eq. (7), do conversor bidirecional, Fig. 2, foi realizado no ambiente do Simulink, com as especificações da Tabela 1 apresentadas na seção anterior IV.

$\mathrm{O}$ vetor de saída $\mathrm{Y}(\mathrm{t})_{\mathrm{dq} 0}$ do modelo matemático Eq. (8) que são as correntes $\mathrm{i}_{\mathrm{d}_{\text {modelo }}}$, $\mathrm{i}_{\mathrm{q} \_ \text {modelo }}$ injetadas na rede elétrica coincidem com as correntes do circuito $\mathrm{i}_{\mathrm{d}}$, $\mathrm{i}_{\mathrm{q}}$. Este resultado pode ser observado na Fig. 9. $\mathrm{O}$ vetor $\mathrm{U}(\mathrm{t})_{\mathrm{dq} 0} \mathrm{de}$ entrada do modelo Eq. (8) consiste nas razões cíclicas $d_{d}, d_{q}, d_{0}$ e nas tensões da rede elétrica $V_{d}, V_{q}, V_{0}$. Mas para o projeto dos quatro compensadores interessa somente as entras $d_{d}, d_{q}$.

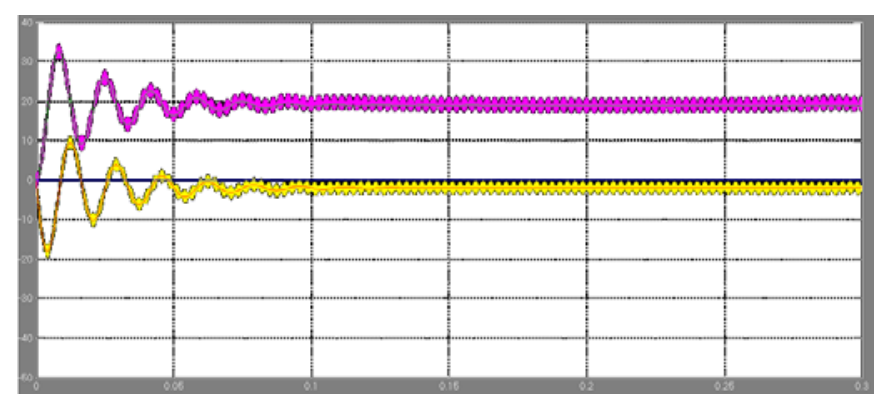

Figura 9. Correntes injetadas na rede do modelo e do circuito inversor.

\subsection{Simulação do Sistema Eólico Conectado à Rede}

Implementou-se o circuito de potência da Fig. 1 no PSIM9.03, gerador PMSG, retificador trifásico e inversor trifásico conectado na rede elétrica e os seus respectivos controles de acordo com a Fig. 3 e a Fig. 4, com as especificações da Tab. 2. 
Tabela 2. Especificações.

\begin{tabular}{|c|c|}
\hline \multicolumn{2}{|c|}{ Gerador PMSG } \\
\hline Potência Nominal da Turbina & $2.0 \mathrm{~kW}$ \\
\hline Tensão de linha RMS/1000 RPM & $380 \mathrm{~V}_{\mathrm{RMS}}$ \\
\hline \multicolumn{2}{|c|}{$\begin{array}{ll}\text { Retificador Trifásico } \\
\end{array}$} \\
\hline Tensão de linha RMS & $380 \mathrm{~V}_{\mathrm{RMS}}$ \\
\hline Frequência de chaveamento $f_{s}$ & $12 \mathrm{KHz}$ \\
\hline Tensão no barramento CC & $350 \mathrm{~V}$ \\
\hline Resistência do filtro $\mathrm{R}_{\mathrm{f}}$ & $0.12 \Omega$ \\
\hline Indutância do filtro $\mathrm{L}_{\mathrm{f}}$ & $2.5 \mathrm{mH}$ \\
\hline Referência de Q* (Pot. Reativa) & 0VAr \\
\hline Referência de P* (Pot. Ativa) & Vel. Vento $\rightarrow$ Lookup table $\rightarrow$ MPPT \\
\hline \multicolumn{2}{|c|}{$\begin{array}{ll}\text { Inversor Trifásico } \\
\end{array}$} \\
\hline Tensão de linha da rede RMS & $127 \mathrm{~V}_{\mathrm{RMS}}$ \\
\hline Frequência da rede & $60 \mathrm{~Hz}$ \\
\hline Tensão no barramento CC & $350 \mathrm{~V}$ \\
\hline Resistência do filtro $R_{f}$ & $0.12 \Omega$ \\
\hline Indutância do filtro $\mathrm{L}_{\mathrm{f}}$ & $2.5 \mathrm{mH}$ \\
\hline Referência de $\mathrm{Q}_{\text {rede }}{ }^{*}$ (Pot. Reativa) & 0VAr \\
\hline Referência de $\mathrm{P}_{\text {rede }}{ }^{*}$ (Pot. Ativa) & Pot. Gerador $\rightarrow$ Retificador \\
\hline
\end{tabular}

Objetivando a comprovação da validade das funções de tranferências $i_{d} / d_{d}$, Eq. (13) e $i_{q} / d_{q}$, Eq. (14) como modelo (planta) dos conversores, no mesmo esquemático do PSIM implementou-se o diagrama de controle da Fig. 5 no controle do retificador e também do inversor.

Implementou-se o diagrama da Fig. 5 para o controle da tensão barramento CC.

As simulações consistiram em degraus aplicados na velocidade do vento seguindo a Tab. 3 . O efeito do degrau de $2 \mathrm{~m} / \mathrm{s}$ em $\mathrm{t}=1$.0seg poderá ser visualizado nas figuras 13 a 18 .

Tabela 3. Variações na velocidade do vento.

\begin{tabular}{c|c|c}
\hline Tempo [seg] & 0.0 & 1.0 \\
\hline Degrau [m/s] & 8 & 2 \\
\hline Veloc. final & 8 & 10 \\
\hline
\end{tabular}

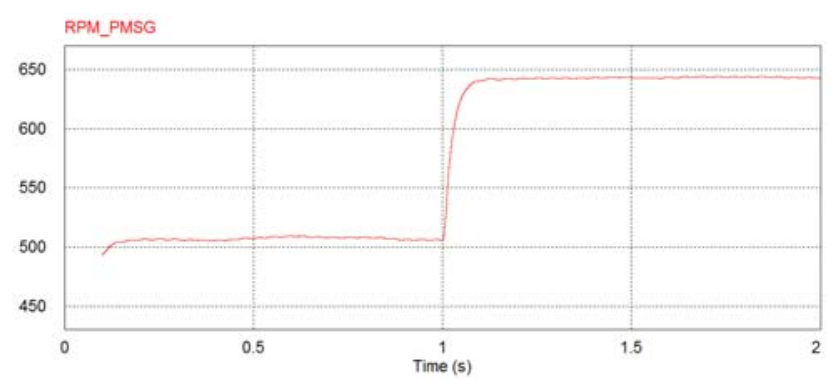

Figura 13. Velocidade do gerador PMSG.

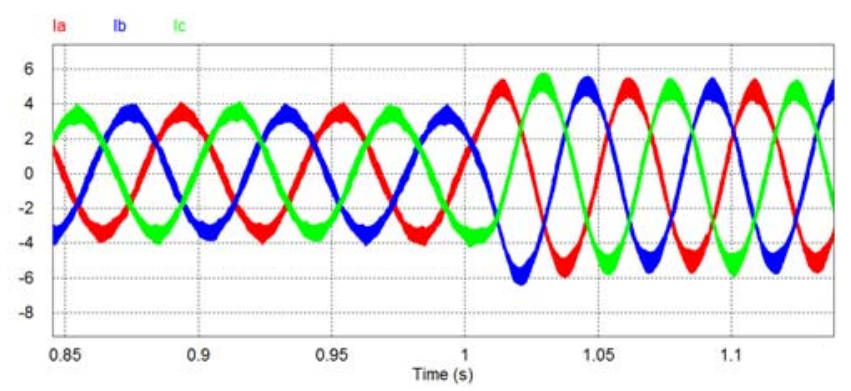

Figura 14. Correntes $I_{a b c}$ na saída do gerador $P M S G$. 


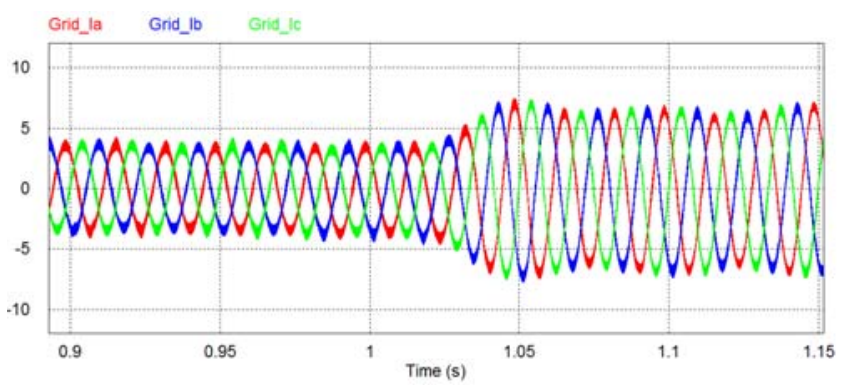

Figura 15. Correntes $I_{a b c}$ injetadas na rede elétrica.

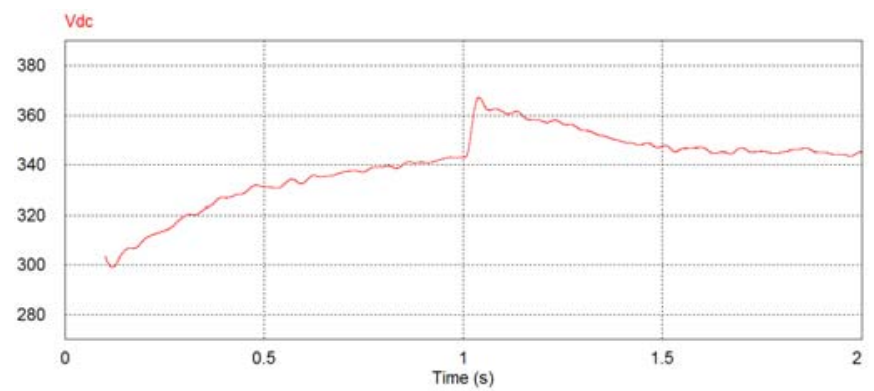

Figura 16. Tensão no barramento $\mathrm{CC}, \mathrm{V}_{\mathrm{dc}}{ }^{*}=350 \mathrm{~V}$.

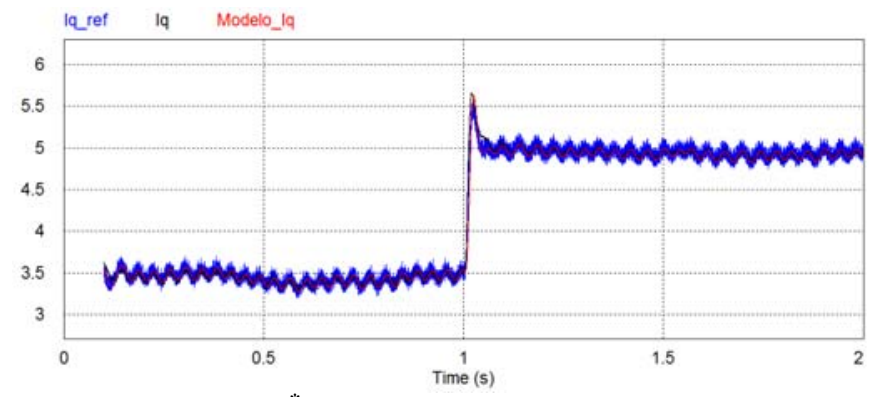

Figura 17. Referência de corrente $i_{q}{ }^{*}, i_{q}$ do circuito, $i_{q}$ do modelo matemático do retificador.

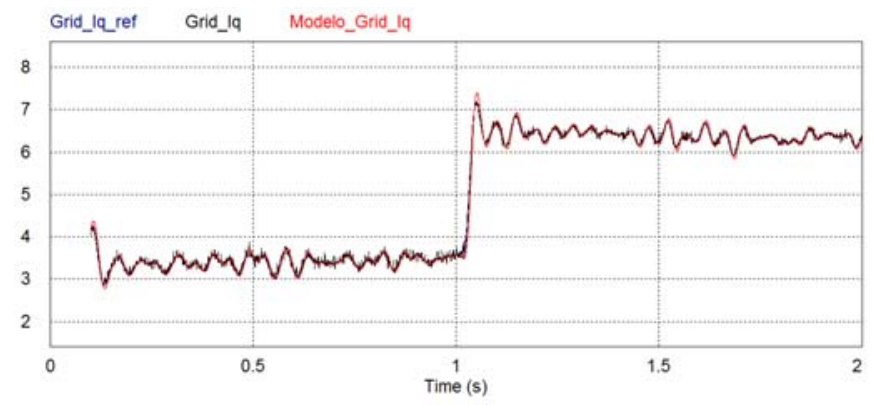

Figura 18. Referência de corrente $i_{\mathbf{q}_{\_} \text {rede }}{ }^{*}, \mathbf{i}_{\mathbf{q} \_ \text {rede }}$ do circuito, $\mathbf{i}_{\mathbf{q} \_ \text {rede }}$ do modelo matemático do inversor.

\section{CONCLUSÕES}

O modelo obtido em espaço de estados em coordenadas dq0 do conversor estático bidirecional forneceu as funções de transferência $i_{d} / d_{d}$ e $i_{q} / d_{q}$ que foram validadas com sucesso em simulação e utilizadas na ferramenta Sisotool do $M a t l a b^{\circledR}$ para projetar os compensadores do tipo PI para os controladores do conversor back-to-back.

A validação das FTs do modelo matemático foram realizadas em simulação no $P S I M^{\circledR}$. As correntes injetadas na rede $i_{\text {d_modelo }} \mathrm{e} i_{\mathrm{q} \text { modelo }}$ foram comparadas com as geradas pelo circuito, obtendo uma perfeita sobreposição e as mesmas seguindo suas respectivas referências $\mathrm{i}_{\mathrm{d}}{ }^{*} \mathrm{e} \mathrm{i}_{\mathrm{q}}{ }^{*}$.

Os compensadores projetados $\mathrm{C} 3$ e $\mathrm{C} 4$ do controle do conversor no lado da rede e utilizados também em $\mathrm{C} 1$ e $\mathrm{C} 2$ no controle do conversor no lado do gerador PMSG apresentaram resultados satisfatórios. As correntes trifásicas geradas e as correntes injetadas na rede foram controladas com êxito de acordo com a potência ativa gerada e ainda rastreando um ponto de máxima operação.

O compensador C5 regulou com êxito a tensão no barramento cc mantendo a tensão próximo do valor de 
referência.

\title{
REFERÊNCIAS
}

Barbi, I., Martins and Denizar C. 2005. Introdução ao estudo dos conversores CC-CA. $2^{\mathrm{a}}$ ed. rev. Florianópolis, Brasil: INEP/UFSC.

Borgono, D. 2005. Tese de Doutorado: “Análise, modelagem e controle de retificadores PWM trifásicos”. Florianópolis, Brasil: INEP/UFSC.

Collier, D. A. F. 2011. Modelagem e controle de retificadores PWM trifásicos conectados a geradores síncronos de imãs permanentes em sistemas de conversão de energia eólica. Florianópolis, Brasil: INEP/UFSC.

Errami, Y. 2012. Control strategy for PMSG wind farm based on mppt and direct power control. IEEE.

Krishnan, R. 2010. Permanent magnet synchronous and brushless DC motor drives. CRC Press.

Li, S. 2009. Conventional and novel control designs for direct driven PMSG wind turbines. Electric Power Systems Research. Brasil: Elsevier.

Muyeen, S. M., Takahashi, R., Ali, M. H. 2007. Transient stability analysis of permanent magnet variable speed synchronous wind generator. Proceeding of international conference on eletrical machines and systems. Coréia do Sul.

Muyeen, S. M., Takahashi, R., Murata, T. 2008. Transient stability enhancement of variable speed wind turbine driven pmsg with rectifier-boost converter-inverter. Proceeding of international conference on eletrical machines and systems. IEEE.

Nascimento, A. C. 2009. Avaliação de estratégias de controle de potência reativa de aerogeradores síncronos em redes elétricas. Brasil: ITEC/PPGEE/UFPA.

Salles, M. B. C. 2009. Modelagem e análise de geradores eólicos de velocidade variável conectados em sistemas de energia elétrica. São Paulo, Brasil: EPUSP.

\section{VARIABLE SPEED WINDTURBINE WITH PMSG CONNECTED TO GRID}

\author{
Maro Jinbo, email: marojinbo@gmail.com ${ }^{1}$ \\ Ghendy Cardoso Junior, email: ghendy@ufsm.br ${ }^{1}$ \\ Felix Alberto Farret (IEEE Member), email: fafarret@gmail.com ${ }^{1}$ \\ Jordan Gustavo Trapp, email: jordantrapp@yahoo.com.br ${ }^{2}$ \\ Edson Ribeiro dos Santos, email:edsonrsmtm@gmail.com ${ }^{3}$ \\ Jawilson Pereira Machado, email: machado@uceff.edu.br ${ }^{4}$ \\ ${ }^{1}$ Federal University of Santa Maria, UFSM - Depart. Electrical Engineering. \\ Avenida Roraima, 1000 Santa Maria/RS. \\ ${ }^{2}$ Federal Institute Sul Rio-Grandense, IFSUL - Departament of Electrical Engineering. \\ Venâncio Aires/RS \\ ${ }^{3}$ Federal University of Fronteira Sul, UFFS - Department of Mathematic. \\ Avenida Fernando Machado, 108 Chapecó/SC. \\ ${ }^{4}$ Faculdade Empresarial de Chapecó (UCEFF) - Department of Environmental and Sanitary Engineering. \\ Rua Lauro Muller, 767 E, Chapecó/SC.
}

\begin{abstract}
This paper presents a VSWT (Variable Speed Wind Turbine) with type generator Permanent Magnet Synchronous Generator PMSG connected to the grid through a converter back-to-back. The modeling tri phase bidirectional converter with three arms IGBTs at State Spaces in the qd0 frame used in the rectification and inversion of the wind system will be validated in simulation. A configuration control PI dq0 coordinates is used to control the converter. Your PI compensator design is demonstrated on simulated Matlab ${ }^{\circledR}$ applied on the generator side converter and also the network. Simulation results using the software PSIM ${ }^{\circledR}$ as both the rectifier inverter are demonstrated to prove the methodologies used and effectiveness of the compensator designed.
\end{abstract}

Keywords: VSWT, PMSG, PI control, grid connection. 